\title{
Prediction of Non-genotoxic Hepatocarcinogenicity Using Chemical-Protein Interactions
}

\author{
Chun-Wei Tung \\ 1 School of Pharmacy, Kaohsiung Medical University, 807, Taiwan \\ ${ }^{2}$ Ph.D. Program in Toxicology, Kaohsiung Medical University, 807, Taiwan \\ cwtung@kmu.edu.tw \\ http://cwtung.kmu.edu.tw
}

\begin{abstract}
The assessment of non-genotoxic hepatocarcinogenicity of chemicals is currently based on 2-year rodent bioassays. It is desirable to develop a fast and effective method to accelerate the identification of potential hepatocarcinogenicity of non-genotoxic chemicals. In this study, a novel method CPI is proposed to predict potential hepatocarcinogenicity of non-genotoxic chemicals. The CPI method is based on chemical-protein interactions and interpretable decision tree classifiers. The interpretable rules generated by the CPI method are analyzed to provide insights into the mechanism and biomarkers of non-genotoxic hepatocarcinogenicity. The CPI method with an independent test accuracy of $86 \%$ using only 1 protein biomarker outperforms the state-ofthe-art methods of gene expression profile-based toxicogenomics using 90 gene biomarkers. A protein ABCC3 was identified as a potential protein biomarker for further exploration. This study presents the potential application of CPI method for assessing non-genotoxic hepatocarcinogenicity of chemicals.
\end{abstract}

Keywords: Non-Genotoxic Hepatocarcinogenicity, Decision Tree, Chemical-Protein Interaction, Interpretable Rule, Toxicology.

\section{Introduction}

Chemical carcinogenesis can be classified into two main categories of genotoxic (mutagenic) and non-genotoxic (non-mutagenic) agents according to the mechanism of action 1, 2]. Several short-term in vitro and in vivo assays have been developed to assess genotoxic agents by measuring DNA damage, mutagenic effects, and chromosomal aberrations [3]. However, due to the complex nature of non-genotoxic agents, the assessment of non-genotoxic hepatocarcinogenicity of chemical compounds is based on 2-year rodent bioassays that is labor-intensive, time-consuming and expensive. There are only 1500 chemicals studied by National Toxicology Program during the past 30 years [4]. It is desirable to develop alternative methods to efficiently prioritize potential non-genotoxic hepatocarcinogenicity of chemicals for further studies. 
Numerous computational models have been developed to predict various toxicity endpoints with reasonably good prediction performance. For example, the quantitative structure-activity relationship (QSAR) models have been extensively used to analyze and predict carcinogenicity [5]. QSAR model aiming to correlate chemical structure information and toxicity endpoints could provide useful information of important structure for toxicity alerts. However, the application of QSAR models for predicting non-genotoxic hepatocarcinogenicity yields a low accuracy of $55 \%$ [9] showing the complexity of non-genotoxic hepatocarcinogenicity.

Recently, toxicogenomics (TGx) correlating gene expression profiles and toxicity endpoints has emerged as important alternative methods. With the power of machine learning methods, TGx performs well in non-genotoxic hepatocarcinogenicity with a test accuracy of $80 \%$,9, 10]. In contrast to traditional 2-year rodent bioassays, TGx methods require much less experimental effort. Generally, published TGx methods select 29 to 120 genes as important biomarkers and require short-term experiments with 5 to 28 days [9, 11, 10]. However, compared to the pure computational method QSAR, TGx methods are still more time-consuming and expensive. Also, chemical-protein interaction (CPI) as an important mechanism for toxicity can not be modeled by TGx methods. The development of fast and accurate methods can largely help the assessment of non-genotoxic hepatocarcinogenicity of chemicals.

The data of CPI information grows very fast in recent years. Benefit from the development of CPI databases, enormous interaction data obtained from databases, experiments and text-mining can be easily accessed from the structured databases including STITCH [12 14], ChemProt [15, 16] and CTD [17]. The databases makes it possible to develop a CPI-based method for analyzing and predicting non-genotoxic hepatocarcinogenicity.

In this study, a CPI based classification method is proposed to analyze and predict non-genotoxic hepatocarcinogenicity of chemicals. Decision tree algorithms capable of generating rule-based knowledge are applied to construct prediction classifiers. The 5 -fold cross-validation and independent test accuracies on training and independent test dataset using only one protein are $82 \%$ and $86 \%$, respectively. The independent test accuracy of the proposed CPI method is better than that of TGx methods requiring 1 to 5-day experiments and 90 biomarkers. This is the first study that utilizes chemical-protein interaction data to predict non-genotoxic hepatocarcinogenicity of chemicals.

\section{Materials and Methods}

\section{$2.1 \quad$ Dataset}

In this study, the development of datasets is based on a liver cancer database NCTRlcdb [18]. In order to demonstrate and compare prediction performances of different methods including CPI, QSAR and toxicogenomics models, only chemicals with existing gene expression data in rat were selected from NCTRlcdb. 
A final dataset consisting of 62 chemicals is utilized to develop and test classifiers for non-genotoxic hepatocarcinogenicity that is developed by Liu et al. [9]. Class labels of either liver carcinogens, carcinogens in other organisms, or noncarcinogens for chemicals were obtained from NCTRlcdb. In order to compare with the QSAR and toxicogenomics models of the previous study [9], the 62 chemicals are divided into a training dataset and an independent test dataset according to the previous study [9]. The training and independent test datasets consisting of 8 positive and 32 negative chemicals and 5 positive and 17 negative chemicals are utilized for training and testing models, respectively.

\subsection{Chemical-Protein Interactions}

Chemical-protein interaction data are obtained from STITCH 3.1 database 13, 14, 12]. STITCH database is an aggregated database of interactions connecting over 300,000 chemicals and 2.6 million proteins from 1133 organisms. The interaction data are obtained from three major sources of experiments, databases and text-mining. The experiment part consists of direct chemical-protein binding data with experimental evidence. The database part contains interaction data from pathway databases. The text-mining data is obtained by extracting information of interactions from literatures using text-mining techniques. Likelihood or relevance scores of interactions are available for each evidence type. An overall score for a given chemical-protein interaction is generated by combining three scores of corresponding evidence types that is available at STITCH [19]. The score is a integer value ranging from 0 (no interaction) to 1000 (strong interaction). Chemical-protein interactions are transferred between species based on the sequence similarity of the proteins [19].

\subsection{Decision Tree Algorithm}

Decision tree algorithms capable of generating interpretable rules based on training data are widely used in various classification and regression problems such as immunogenic peptides [20], ubiquitination sites [21], gamma-turn types [22] and protein subnuclear localization [23]. In this study, the decision tree method C5.0 is applied to construct decision tree classifiers and derive interpretable rules based on chemical-protein interaction profile for classifying non-genotoxic hepatocarcinogenicity. C5.0 is an improved version of C4.5 with smaller trees and less computation time [24]. The implementation of C5.0 used in this study is the R package C50 [25].

The construction of a decision tree is described as follows. First, information gain is utilized to rank features. Second, the top-ranking features are iteratively appended as nodes to split data into subsets. The tree growing process stops when the data subset in each leaf node belongs to the same class. The fullygrown tree is prone to over-fit the training data. Therefore, a pruning process is applied to reduce the tree size by replacing a subtree with a leaf node to avoid over-fitting problems. The pruning process is based on a default threshold 
value of $25 \%$ confidence. The samples in the leaf node are the covered samples of this rule. The class label of a leaf node is determined by using majority rule. The samples with a relative small size in the leaf node are regarded as misclassified samples. The final decision tree can directly generate if-then rules where one leaf node corresponds to one rule.

\subsection{Performance Measurement}

To evaluate classifiers for their prediction performance, the widely used 5 -fold cross-validation method is applied. Four measurements were applied to evaluate classifiers including sensitivity, specificity, accuracy and Matthews correlation coefficient (MCC) defined as follows:

$$
\begin{gathered}
\text { Sensitivity }=\frac{T P}{T P+F N}, \\
\text { Specificity }=\frac{T N}{T N+F P}, \\
\text { Accuracy }=\frac{T P+T N}{T P+F P+F N+T N}, \\
\mathrm{MCC}=\frac{T P \times T N-F P \times F N}{\sqrt{(T P+F P)(T P+F N)(T N+F P)(T N+F N)}},
\end{gathered}
$$

where $T P, F P, F N$ and $T N$ are the numbers of true positives, false positives, false negatives and true negatives, respectively. In this work, accuracy is used as major indicator for estimating the performance of classifiers.

\section{Results and Discussion}

\subsection{Classification Performance on Training Dataset}

The proposed CPI method is based on information of chemical-protein interactions. The chemical of $N, N^{\prime}$-diphenyl-p-phenylenediamine without a corresponding record in STITCH database is excluded from the following analyses. The chemicals in the training dataset is firstly transformed to 4 matrixes of chemical-protein interaction scores obtained from combined scores, databases, experiments and text-mining. Only the CPI information of Rattus norvegicus is used because the hepatocarcinogenic annotation of the 62 chemicals is based on rat and mouse. In order to provide better insights into protein biomarkers of non-genotoxic hepatocarcinogenicity, the decision tree algorithm C5.0 is applied to generate human interpretable rules based on training datasets for further confirmation. 
Table 1. Cross-validation performance

\begin{tabular}{lcccc}
\hline Model type & Classifier & Feature selection & $\begin{array}{c}\text { Number of } \\
\text { selected features }\end{array}$ & $\begin{array}{c}5 \text {-CV } \\
\text { accuracy }\end{array}$ \\
\hline CPI & C5.0 & Information gain & 1 & 0.82 \\
QSAR $^{*}$ & NCC & Wrapper-based mRMR & 15 & 0.76 \\
TGx (1-day) $^{*}$ & NCC & Wrapper-based mRMR & 90 & 0.87 \\
TGx (3-day) $^{*}$ & NCC & Wrapper-based mRMR & 90 & 0.87 \\
TGx (5-day) & NCC & Wrapper-based mRMR & 90 & 0.90 \\
\hline
\end{tabular}

${ }^{*}$ Model performance from Liu et al [9]

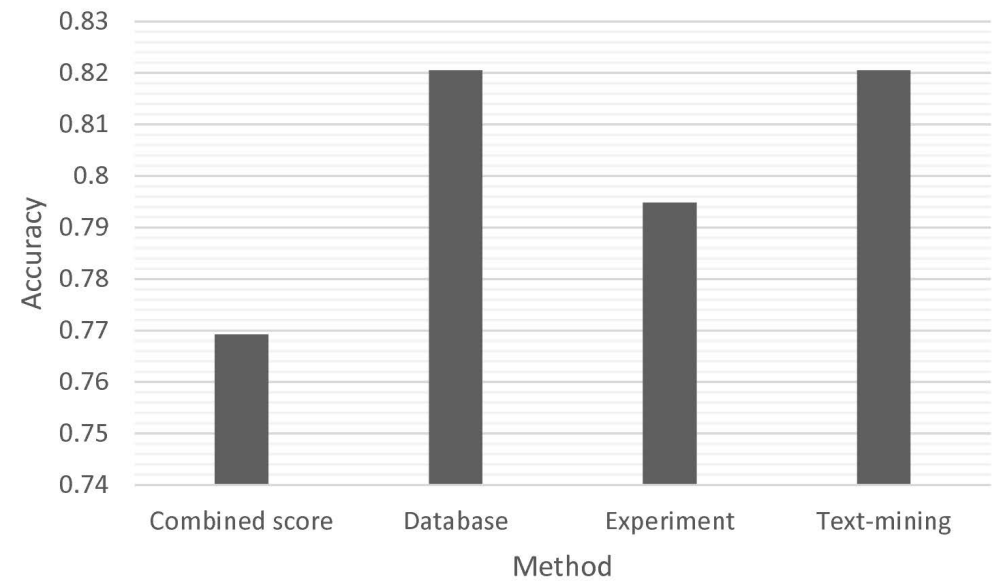

Fig. 1. Five-fold cross-validation performance of CPI method using various scores as features

To evaluate the classification performance of the CPI method, a 5-fold crossvalidation $(5-\mathrm{CV})$ is applied to the training dataset consisting of 7 positive and 31 negative chemicals. In the 5 -CV, the training dataset is firstly divided into 5 folds with nearly equal number of chemicals. For each validation fold $f$ of the 5-CV, C5.0 is applied to select important features for constructing a decision tree classifier based on the remaining 4 folds and evaluate its performance on the validation fold. The 5-CV performances of the CPI method for 4 matrixes are shown in Fig. 1. The CPI scores obtained from databases and text-mining perform best with the same accuracy of $82.05 \%$. The accuracy of experimentderived CPI scores is slightly worse with an accuracy of $79.49 \%$. The CPI scores obtained by combining three data sources of databases, experiments and textmining perform worst with an accuracy of $76.92 \%$.

The information obtained from databases including metabolic pathway information is used for the following analysis that could be more useful than information from text-mining because chemical metabolites might be more toxic 
than parent chemicals. Table 1 shows the detailed 5-CV performance of the CPI method and published QSAR and TGx methods [9]. All the QSAR and TGx methods are based on a nearest-centroid classifier (NCC) and a wrapper-based feature selection based on the ranking calculated by a minimum redundancymaximum relevancy (mRMR) [26]. The CPI method utilizing a simple and human interpretable classifier C5.0 shows good accuracy of 0.82 that is better than the QSAR model. Although TGx models show better accuracies than CPI, its feature selection method is a wrapper-based method that is more likely to overfit the training dataset and overestimate its prediction performance. Additionally, the proposed CPI method utilizes only 1 feature for each fold with interpretable rules that is much smaller than the QSAR and TGx models requiring 15 and 90 features without interpretable rules, respectively. The selected features will be discussed in the next section.

\subsection{Feature Selection of Important Proteins}

For each fold of the 5-fold cross-validation, C5.0 select important features for constructing a decision tree classifier. The interpretation of the decision tree classifier can provide better understanding of non-genotoxic hepatocarcinogenicity. The important features of the five decision trees are shown in Table 2 with a usage value showing the percentage of covered chemicals.

Due to the simple decision tree created for each fold with only one protein, all the usage values are $100 \%$. The ABCC3 protein is identified as an important protein in two folds (40\%) showing its critical role in non-genotoxic hepatocarcinogenicity. ABCC3 (ATP-binding cassette, subfamily C (CFTR/MRP), member 3 ) is a member of the superfamily of ATP-binding cassette (ABC) transporters that transports various molecules across membranes. ABCC3, also known as the canalicular multispecific organic anion transporter 2, exhibits drug transmembrane transporter activity that is critical for drug transport, multidrug resistance and bile acid transport pathways. The rule associated with ABCC3 is 'IF a chemical interacts with ABCC3 THEN it is a hepatocarcinogenic chemical'.

The protein MPO is a myeloperoxidase with peroxidase activity and is found in extracellular space, mitochondrion and secretory granule. Previous studies have reported possible roles of oxidative stress on carcinogenicity [27, 28]. MPO as an antioxidant enzyme is able to detoxify the reactive oxygen species (ROS) of oxidative stress. Chemicals interacting with MPO could interrupt the detoxicification process and lead to carcinogenicity.

Serotransferrin (TF) exhibiting the activity of binding and transmembrane transporter of ferric iron is identified in the third fold. Iron in its free form is carcinogenic unless it is bound to ferritin or transferrin [29 31]. The carcinogenicity of TF-interacting chemicals might be caused by their interference with the loading of iron.

The protein RB1 of retinoblastoma 1 associated with retinoblastoma is found to be involved in the non-genotoxic hepatocarcinogenicity 32]. RB1 is a tumor suppressor protein for preventing excessive cell growth by inhibiting cell cycle progression [33]. The dysfunction of RB1 could cause carcinogenicity. 
Table 2. Important proteins identified from 5-fold cross-validation

\begin{tabular}{cllr}
\hline Fold & Name & Description & Usage \\
\hline 1 & ABCC3 & ATP-binding cassette, subfamily C (CFTR/MRP), member 3 & $100 \%$ \\
2 & MPO & Myeloperoxidase & $100 \%$ \\
3 & TF & Serotransferrin & $100 \%$ \\
4 & RB1 & Retinoblastoma 1 & $100 \%$ \\
5 & ABCC3 & ATP-binding cassette, subfamily C (CFTR/MRP), member 3 & $100 \%$ \\
\hline
\end{tabular}

Altogether, the identified proteins and functions are consistent with possible mechanisms of non-genotoxic carcinogenicity reported by previous studies, including modulation of metabolic enzymes, induction of peroxisome proliferation and alteration of intercellular communication [34 37 ].

\subsection{Independent Test}

To further evaluate the prediction ability of the CPI method, the proposed CPI method is applied to train a decision tree classifier based on the training dataset and predict the independent test dataset consisting of 20 chemicals. A search of the chemical of lead(iv) acetate in STITCH database leads to the record of lead(ii) acetate of the same CPI profiles. To avoid overestimate the prediction performance of the CPI method, the chemical of lead(iv) acetate is excluded for the following analysis. The same as the $5-\mathrm{CV}$ with 1 protein selected for each fold, only 1 protein is selected to construct a decision tree classifier. The decision tree shown in Fig. 2 represents a very simple rule of 'IF a chemical interacts with ABCC3 THEN it is a hepatocarcinogenic chemical'. The rule is surprisingly simple and correctly predict $90 \%$ chemicals in the training dataset with only 4 misclassified chemicals. All 31 non-hepatocarcinogenic chemicals do not interact with protein ABCC3. Fifty percent of hepatocarcinogenic chemicals interact with ABCC3. Chemicals interact with ABCC3 might interfere the normal function of chemical transportation.

Table 3. Independent test performance

\begin{tabular}{lccccc}
\hline Model type & $\begin{array}{c}\text { Number of } \\
\text { selected features }\end{array}$ & Accuracy & Sensitivity & Specificity & MCC \\
\hline CPI & 1 & 0.86 & 0.40 & 1.00 & 0.580 \\
QSAR $^{*}$ & 15 & 0.55 & 0.20 & 0.65 & -0.138 \\
TGx (1-day) $^{*}$ & 90 & 0.77 & 0.40 & 0.88 & 0.307 \\
TGx (3-day) $^{*}$ & 90 & 0.77 & 0.20 & 0.94 & 0.206 \\
TGx (5-day) $^{*}$ & 90 & 0.82 & 0.60 & 0.88 & 0.482 \\
\hline
\end{tabular}

${ }^{*}$ Model performance from Liu et al [9]

To demonstrate the prediction ability of the proposed CPI method, the decision tree classifier is applied to predict chemicals in the independent test dataset. The prediction results are shown in Table 3. The simple decision tree classifier 


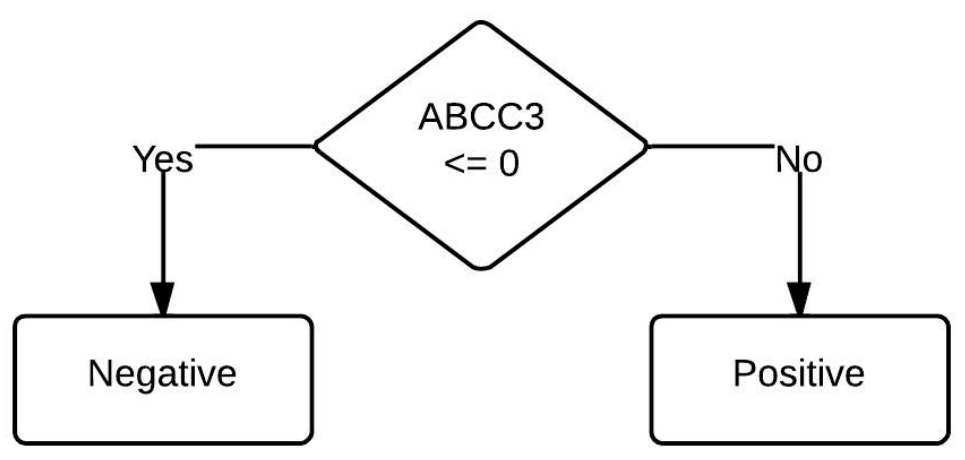

Fig. 2. The constructed decision tree based on the training dataset

of CPI performs very well with an accuracy of $86 \%$ that is better than QSAR, 1-day TGx, 3-day TGx and 5-day TGx models with accuracies of 55\%, 77\%, $77 \%$ and $82 \%$. The MCC value as a more objective evaluation of performance for unbalanced data is also used to evaluate prediction performance. The MCC values for CPI, QSAR, 1-day TGx, 3-day TGx and 5-day TGx models are 0.580, $-0.138,0.307,0.206$ and 0.482 , respectively. The CPI method with highest MCC value performs best.

The wrapper-based feature selection method used in the previous study [9] might overestimate the $5-\mathrm{CV}$ accuracies on the training dataset and result in a large decrease in prediction accuracies on the independent test dataset. The proposed CPI method utilizing only a single feature with human interpretable rules outperforms QSAR and TGx methods showing that chemical-protein interactions are useful for predicting non-genotoxic hepatocarcinogenicity of chemicals.

\section{Conclusions}

Alternative methods for assessing non-genotoxic hepatocarcinogenicity of chemicals could save a lot of time and money and reduce the consumption of animals for testing. The traditional QSAR model is not effective in discrimination of hepatocarcinogenicity of non-genotoxic chemicals [9] showing the complex nature of non-genotoxic hepatocarcinogenicity involving many genes and proteins. In contrast to chemical structure-based QSAR models, TGx methods based on gene expression-profiles can model the complex mechanism in the transcriptomics level and perform better than the QSAR model [9].

The mechanism of action of non-genotoxic hepatocarcinogenicity might involve complex regulations of proteins and chemicals. Hence, the application of CPI data for developing classifiers is expected to outperform QSAR and TGx methods. This study presents a novel CPI-based method and demonstrates the effectiveness of biomarker identification and superior prediction performance. The utilization of simple decision tree algorithms generates human-interpretable rules for better understanding of key proteins for non-genotoxic hepatocarcinogenicity. 
The identified proteins could serve as important biomarkers for further applications to the assessment of non-genotoxic hepatocarcinogenicity of chemicals. Compared to TGx methods requiring assessment of 100 gene expression values and 5 to 28-day experiments, the identified single biomarker could be more cost-effective and time-saving. Future works include the application of advanced machine learning algorithms such as support vector machines and collection of a larger dataset for improving prediction accuracy.

Acknowledgement. The authors would like to acknowledge the financial support from National Science Council of Taiwan (NSC 101-2311-B-037-001-MY2) and Kaohsiung Medical University Research Foundation (KMU-Q110015 and KMU-ER-013).

\section{References}

1. Hayashi, Y.: Overview of genotoxic carcinogens and non-genotoxic carcinogens. Exp. Toxicol. Pathol. 44, 465-471 (1992)

2. Melnick, R.L., Kohn, M.C., Portier, C.J.: Implications for risk assessment of suggested nongenotoxic mechanisms of chemical carcinogenesis. Environ. Health Perspect. 104 (suppl. 1), 123-134 (1996)

3. Weisburger, J.H., Williams, G.M.: The distinction between genotoxic and epigenetic carcinogens and implication for cancer risk. Toxicol. Sci. 57, 4-5 (2000)

4. Gold, L.S., Manley, N.B., Slone, T.H., Rohrbach, L., Garfinkel, G.B.: Supplement to the carcinogenic potency database (cpdb): results of animal bioassays published in the general literature through 1997 and by the national toxicology program in 1997-1998. Toxicol. Sci. 85, 747-808 (2005)

5. Kar, S., Deeb, O., Roy, K.: Development of classification and regression based qsar models to predict rodent carcinogenic potency using oral slope factor. Ecotoxicol. Environ. Saf. 82, 85-95 (2012)

6. Kar, S., Roy, K.: First report on development of quantitative interspecies structurecarcinogenicity relationship models and exploring discriminatory features for rodent carcinogenicity of diverse organic chemicals using oecd guidelines. Chemosphere 87, 339-355 (2012)

7. Tanabe, K., Kurita, T., Nishida, K., Lucic, B., Amic, D., Suzuki, T.: Improvement of carcinogenicity prediction performances based on sensitivity analysis in variable selection of svm models. SAR QSAR Environ. Res. (2013)

8. Yuan, J., Pu, Y., Yin, L.: Qsar study of liver specificity of carcinogenicity of nnitroso compounds. Ecotoxicol. Environ. Saf. 84, 282-292 (2012)

9. Liu, Z., Kelly, R., Fang, H., Ding, D., Tong, W.: Comparative analysis of predictive models for nongenotoxic hepatocarcinogenicity using both toxicogenomics and quantitative structure-activity relationships. Chem. Res. Toxicol. 24, 10621070 (2011)

10. Yamada, F., Sumida, K., Uehara, T., Morikawa, Y., Yamada, H., Urushidani, T., Ohno, Y.: Toxicogenomics discrimination of potential hepatocarcinogenicity of non-genotoxic compounds in rat liver. J. Appl. Toxicol. (2012)

11. Uehara, T., Hirode, M., Ono, A., Kiyosawa, N., Omura, K., Shimizu, T., Mizukawa, Y., Miyagishima, T., Nagao, T., Urushidani, T.: A toxicogenomics approach for early assessment of potential non-genotoxic hepatocarcinogenicity of chemicals in rats. Toxicology 250, 15-26 (2008) 
12. Kuhn, M., von Mering, C., Campillos, M., Jensen, L.J., Bork, P.: Stitch: interaction networks of chemicals and proteins. Nucleic Acids Res. 36, D684-D688 (2008)

13. Kuhn, M., Szklarczyk, D., Franceschini, A., Campillos, M., von Mering, C., Jensen, L.J., Beyer, A., Bork, P.: Stitch 2: an interaction network database for small molecules and proteins. Nucleic Acids Res. 38, D552-D556 (2010)

14. Kuhn, M., Szklarczyk, D., Franceschini, A., von Mering, C., Jensen, L.J., Bork, P.: Stitch 3: zooming in on protein-chemical interactions. Nucleic Acids Res. 40, D876-D880 (2012)

15. Kim Kjaerulff, S., Wich, L., Kringelum, J., Jacobsen, U.P., Kouskoumvekaki, I., Audouze, K., Lund, O., Brunak, S., Oprea, T.I., Taboureau, O.: Chemprot-2.0: visual navigation in a disease chemical biology database. Nucleic Acids Res. 41, 464-469 (2013)

16. Taboureau, O., Nielsen, S.K., Audouze, K., Weinhold, N., Edsgard, D., Roque, F.S., Kouskoumvekaki, I., Bora, A., Curpan, R., Jensen, T.S., Brunak, S., Oprea, T.I.: Chemprot: a disease chemical biology database. Nucleic Acids Res. 39, D367D372 (2011)

17. Mattingly, C.J., Colby, G.T., Forrest, J.N., Boyer, J.L.: The comparative toxicogenomics database (ctd). Environ. Health Perspec.t 111, 793-795 (2003)

18. Young, J., Tong, W., Fang, H., Xie, Q., Pearce, B., Hashemi, R., Beger, R., Cheeseman, M., Chen, J., Chang, Y.C., Kodell, R.: Building an organ-specific carcinogenic database for sar analyses. J. Toxicol. Environ. Health A 67, 1363-1389 (2004)

19. von Mering, C., Jensen, L.J., Snel, B., Hooper, S.D., Krupp, M., Foglierini, M., Jouffre, N., Huynen, M.A., Bork, P.: String: known and predicted protein-protein associations, integrated and transferred across organisms. Nucleic Acids Res. 33, D433-D437 (2005)

20. Tung, C.W., Ho, S.Y.: Popi: predicting immunogenicity of mhc class i binding peptides by mining informative physicochemical properties. Bioinformatics 23, 942-949 (2007)

21. Tung, C.W., Ho, S.Y.: Computational identification of ubiquitylation sites from protein sequences. BMC Bioinformatics 9, 310 (2008)

22. Liaw, C., Tung, C.W., Ho, S.J., Ho, S.Y.: Sequence-based prediction of gamma-turn types using a physicochemical property-based decision tree method. Proceeding of World Academy of Science, Engineering and Technology 41, 898-902 (2010)

23. Huang, W.L., Tung, C.W., Ho, S.W., Ho, S.Y.: Proloc-rgo: Using rule-based knowledge with gene ontology terms for prediction of protein subnuclear localization. In: IEEE Symposium on Computational Intelligence in Bioinformatics and Computational Biology, CIBCB 2008, pp. 201-206. IEEE (2008)

24. Quinlan, J.: C4. 5: programs for machine learning. Morgan kaufmann (1993)

25. Kuhn, M., Weston, S.: code for C5.0 by R. Quinlan, N.C.C.: C50: C5.0 Decision Trees and Rule-Based Models (2012); R package version 0.1.0-013

26. Ding, C., Peng, H.: Minimum redundancy feature selection from microarray gene expression data. J. Bioinform. Comput. Biol. 3, 185-205 (2005)

27. Kryston, T.B., Georgiev, A.B., Pissis, P., Georgakilas, A.G.: Role of oxidative stress and dna damage in human carcinogenesis. Mutat. Res. 711, 193-201 (2011)

28. Ziech, D., Franco, R., Georgakilas, A.G., Georgakila, S., Malamou-Mitsi, V., Schoneveld, O., Pappa, A., Panayiotidis, M.I.: The role of reactive oxygen species and oxidative stress in environmental carcinogenesis and biomarker development. Chem. Biol. Interact. 188, 334-339 (2010)

29. Ponka, P., Beaumont, C., Richardson, D.R.: Function and regulation of transferrin and ferritin. Semin. Hematol. 35, 35-54 (1998) 
30. McCord, J.M.: Iron, free radicals, and oxidative injury. Semin. Hematol. 35, 5-12 (1998)

31. Linn, S.: Dna damage by iron and hydrogen peroxide in vitro and in vivo. Drug Metab. Rev. 30, 313-326 (1998)

32. Gill, J.H., Brickell, P., Dive, C., Roberts, R.A.: The rodent non-genotoxic hepatocarcinogen nafenopin suppresses apoptosis preferentially in non-cycling hepatocytes but also elevates cdk4, a cell cycle progression factor. Carcinogenesis 19, 1743-1747 (1998)

33. Weinberg, R.A.: The retinoblastoma protein and cell cycle control. Cell 81, 323-330 (1995)

34. Butterworth, B.E., Bogdanffy, M.S.: A comprehensive approach for integration of toxicity and cancer risk assessments. Regul. Toxicol. Pharmacol. 29, 23-36 (1999)

35. Nguyen-Ba, G., Vasseur, P.: Epigenetic events during the process of cell transformation induced by carcinogens (review). Oncol. Rep. 6, 925-932 (1999)

36. Silva Lima, B., Van der Laan, J.W.: Mechanisms of nongenotoxic carcinogenesis and assessment of the human hazard. Regul. Toxicol. Pharmacol. 32, 135-143 (2000)

37. Williams, G.M., Iatropoulos, M.J., Weisburger, J.H.: Chemical carcinogen mechanisms of action and implications for testing methodology. Exp. Toxicol. Pathol. 48, 101-111 (1996) 